Ann. Zootech., I977, 26 (3), 395-4II.

\title{
Collecte totale des contenus intestinaux chez le Porc Automatisation de l'échantillonnage et de la restitution à l'animal pour une étude cinétique de la digestion ${ }^{1}$ )
}

\author{
L. P. BORGIDA ct J.-P. LAPLACE * \\ avec la collaboration technique de C.Germain et Monique Diez \\ Laboratoive de Technologie des Aliments des Animanx, \\ * Laboratoire de Physiologic de la Nutrition, \\ Centre national de Recherches zootechniques, I.N.R.A., \\ 78350 Jouy-en-Josas (France)
}

\begin{abstract}
Résumé
L'intérêt potentiel du développement d'un système automatisé de collecte totale, échantillonnage et restitution des contenus digestifs, est analysé dans la perspective de l'animal monogastrique et plus spécialement de l'expérimentation chez le Porc. Cette réflexion repose sur l'étude bibliographique des travaux d'ordre méthodologique réalisés chez les Ruminants, pour évaluer la technique de collecte totale et celle des marqueurs, face aux particularités physiologiques et comportementales du Porc.

Compte tenu des conclusions ainsi atteintes, la recherche des principes de fonctionnement d'un système automatisé do collecte des digesta est relatée. Leur mise en ceuvre a conduit à réaliser un appareillage totalement automatique. L'analyse expérimentale de ses avantages et inconvénients spécifiques amène enfin à l'élaboration d'un système semi-automatique qui s'avère le mieux adapté à l'enregistrement chez le Porc. Cette semi-automatisation ne permet plus la parfaite simultanéité de la collecte, de l'échañtillonnage et de la réintroduction qu'apporte l'automatisme total; mais elle permet une parfaite représentativité de l'échantillonnage et une précision meilleure (de l'ordre de 2 à $3 \mathrm{p}$. Ioo) des mesures de volume, et partant une meilleure estimation de la digestion de tel ou tel constituant alimentaire.

Les systèmes décrits resteni assurément perfectibles. L'éventualité d'un asservissement de diverses manœuvres à une mesure électromagnétique du débit des digesta est envisagée.
\end{abstract}

\section{Introduction}

L'une des deux questions essentielles qui se posent pour l'étude de l'absorption de tel ou tel nutriment concerne la nature, la distribution et l'origine des substances présentes dans l'intestin (RERAT, Corring, Laplace, I976). La simple différence entre les quantités ingérée et excrétée d'un nutriment ne reflète pas

(I) Requests for reprints to J. P. IAAPLACE. 
nécessairement l'absorption par la paroi intestinale. Aussi, la connaissance de la répartition des processus de digestion au long du tractus gastro-intestinal est primordiale pour la compréhension des phénomènes d'absorption (FAICHNEY, 1975) Une collecte quantitative des contenus digestifs, chez l'animal porteur de canules réentrantes, autorise l'étude cinétique du passage et de la transformation biochimique des digesta, et celle de la disparition des produits de la digestion.

Mais s'il est en théorie aisé de réaliser, dans ces conditions de collecte totale, un échantillonnage représentatif, diverses difficultés doivent être surmontées : éventualité d'un effet lié à la présence même des canules, perturbation possible des processus physiologiques par la dérivation temporaire des digesta, modification bien connue du débit à la canule proximale en fonction du rythme de restitution des effluents collectés. Nous avons déjà envisagé l'influence perturbatrice de la présence des canules réentrantes (LAPLACE, I972-I975). Il y a là une véritable certitude dans le cas de la fistulation de l'iléon terminal chez le Porc (LAPLACE et BORGIDA, I976). Il ne semble pas qu'il en soit de même lors de fistulation duodénale, sous réserve d'une adaptation à la topographie viscérale de l'espèce (LAPLACE, TOMASSONE, I970), mais cet aspect particulier fait actuellement l'objet de travaux expérimentaux.

C'est pour répondre aux deux autres difficultés que nous avons tenté de développer un système automatisé de collecte, échantillonnage et restitution des digesta à l'usage du Porc. Mais il paraît utile de situer ici l'intérêt de cette approche par rapport aux autres techniques couramment employées chez l'animal, en l'envisageant du point de vue des Monogastriques, en particulier du Porc, à partir des données disponibles chez les Ruminants. Ce n'est en effet que chez ces derniers qu'ont été réalisées de multiples études méthodologiques.

\section{I. - Intérêt potentiel de l'automatisation des opérations lors de collecte totale des digesta}

Schématiquement, deux méthodologies permettent l'étude du débit des digesta en tel ou tel point du tube digestif, et d'une façon générale du déroulement de la digestion. L'une consiste à extérioriser le flot des digesta en vue d'une mesure directe, et utilise la fistulation réentrante; l'autre fournit par le calcul l'estimation de ce débit, par référence à des marqueurs indigestibles (KoTB et LuCKEY, I972).

\section{I. - Usage des marqueurs}

Cette seconde méthodologie a été clairement développée et critiquée par FAICHNEy (I975) dans le cas des Ruminants. Elle peut être utilisée sous quatre formes résultant des combinaisons de deux modes d'administration (apport continu ou dose unique) et de deux modes de prélèvement (prélèvement intermittent des contenus en un site choisi ou prélèvement de tout le tube digestif plein au moment de l'abattage). L'usage combiné de la dose unique de marqueur et de l'abattage n'est guère utilisable que chez les petites espèces telles que le Lapin (LAPLACE et LEBAS, I975). L'association de la perfusion continue du traceur et de l'abattage est plus spécialement intéressante pour l'étude des mouvements d'eau et d'électrolytes.

Des deux techniques utilisant le principe de prélèvements intermittents, l'une (apport continu) s'avère particulièrement intéressante pour la mesure des débits 
de digesta chez les Ruminants, et l'autre (dose unique) pour la mesure des temps de rétention ou des rythmes d'écoulement hors de compartiments réservoirs. Mais toutes les deux, quoique bénéficiant de l'avantage de ne nécessiter qu'une canule simple comme toutes les techniques de marqueurs, et contrairement aux Ruminants, conviennent très mal au Porc. En effet un prélèvement discontinu, par exemple de $5 \mathrm{mn}$ toutes les 2 heures (EHounsou, I976) a, chez ce dernier, fort peu de chance de représenter un volume significatif au delà de la troisième heure après le repas en raison de l'intermittence des évacuations gastriques (AUFfray, Martinet, Rerat, ig67 - Laplace, Tomassone, i97o). L'inadéquation de ces techniques résulte de la discontinuité liée aux "repas " peu nombreux chez le Monogastrique (du fait de la conjugaison de l'organisation du comportement alimentaire propre à l'espèce et de l'influence des horaires de distribution) par opposition au régime d'écoulement beaucoup plus régulier dans l'intestin des polygastriques.

\section{2. - Fistulation réentrante}

C'est donc préférentiellement vers la méthodologie fondée sur l'usage de canules réentrantes que s'oriente l'étude de la digestion chez le Porc. L'analyse critique de cette méthodologie n'a guère été effectuée que chez les Ruminants. Mais toutes les données recueillies et discutées par Macrae (I975) montrent que des collectes brèves de l'ordre de $\mathrm{I} 2$ à $24 \mathrm{~h}$ sont responsables de réductions du débit de digesta de l'ordre de I5 à 30 p. I Oo, avec compensation ultérieure. Ilsemble même selon les résultats de EHouinsou (I976) que la perturbation du transit persiste durant $48 \mathrm{~h}$ environ, en raison d'une inadaptation de l'animal à la situation de collecte totale, toute anomalie de réintroduction étant écartée. Cette perturbation, postulée sur la base de grandes variations de la concentration des marqueurs dans les contenus digestifs, est confirmée lors de mesure directe du transit de la matière sèche.

Il y a donc là, outre la plus grande complication des manipulations liées à des canules réentrantes, un élément en défaveur de cette méthodologie. Mais il se trouve largement compensé par le fait que l'échantillonnage qui peut être réalisé lors de prélèvement continu (collecte totale) est beaucoup plus représentatif des contenus digestifs. On évite en effet d'échantillonner un contenu particulièrement riche en solides, ou inversement très liquide, comme ce peut être le cas en prélèvement discontinu. Or nous avons vu l'importance chez le Porc de ce phénomène normal de séparation des phases liquide et solide (LAPLACE et TOMassone, I970 - LAPLACE, I 975).

\section{3. - Vers un choix raisonné}

En somme, malgré l'incertitude pesant sur la technique de fistulation réentrante (effet de la présence des canules) et sa plus grande complexité de mise en œuvre, c'est bien la collecte totale des contenus, dans le cadre d'un prélèvement continu, qui est à rechercher chez le Porc principalement en raison de l'ingestion de repas peu nombreux, et en raison d'une qualité d'échantillonnage précieuse dans une étude de la digestion de constituants alimentaires soumis à évolution rapide (amidon, matières azotées...). Il convient donc de parer aux perturbations décrites chez les Ruminants lors de collecte totale à court terme puisque l'analyse du transit gastro-intestinal d'un repas d'épreuve chez le Porc portera sur Io à I2 $\mathrm{h}$. 
Deux solutions sont utilisables qui ont été envisagées par Macrae (I975). La correction des débits mesurés en collecte totale par l'usage de marqueurs en est une. Mais elle ne sera pas nécessairement adéquate en raison du risque de migration non simultanée du traceur. L'automatisation des opérations de prélèvement, échantillonnage et réintroduction constitue la seconde, et sans doute la plus intéressante, des deux possibilités.

\section{4. - Les essais d'automatisation}

Indépendamment du problème de la durée des enregistrements et de la contrainte qu'elle implique, la collecte totale des contenus digestifs, avec échantillonnage et restitution automatisés, apparaît donc comme la technique de choix pour une étude cinétique de la digestion chez un monogastrique tel que le Porc. Cet intérêt potentiel est tel que divers appareillages ont été proposés dans le cas des Ruminants pour lesquels d'autres méthodologies sont pourtant satisfaisantes.

Ainsi Evans, Axford et Offer (I97I) estimant nécessaire une correction des débits dans le cas de collecte manuelle, ont proposé (AxFord, Evans et OFFER, I97I) un appareillage automatique mis en œuvre chez le Mouton. Parallèlement, Thivend et Poncet (I97I) ont développé un autre type d'appareillage, à l'usage du Veau et du Mouton, avec tambour rotatif de collecte ; TAYlor, Weller et REID (I97I) ont décrit un système ingénieux, porté par le Mouton, avec réservoir d'homogénéisation au niveau de la canule elle-même et dispositif d'échantillonnage sur le principe de la seringue. Puis, CoRse et Sutton qui soulignaient en I97 r la difficulté d'interprétation des résultats obtenus chez les Ruminants lors d'usage de marqueurs, ont dressé (CORSE, 1974) un bilan des équipements semi-automatiques ou automatiques; sur cette base, ils ont élaboré pour la Vache un système dérivé de celui d'AXFord Evans et OfFer (I97I) dont l'usage (Sutton, Yousser et OLDHAM, I976) fournit de meilleurs résultats qu'une correction des débits par l'usage de marqueurs.

Selon Macrae (I975) il est encore trop tôt pour procéder à l'heure actuelle à une évaluation critique complète de l'usage de ces divers systèmes pour les Ruminants. En l'absence totale d'expérience chez les Monogastriques, on peut ajouter, aux motivations du choix déjà évoquées, que l'automatisation apporte l'avantage de la simultanéité des manœuvres de collecte - réintroduction, de leur reproductibilité, et de la continuité d'un échantillonnage rigoureux sans temporisation excessive (ZEBROWSKA et al., I975 : prélèvement d'un aliquote de $5 \mathrm{p}$. Ioo des digesta collectés en $30 \mathrm{mn}$ - HoLmes, BAyLEy et HoRney, I 974 : prélèvement d'un aliquote de ro p. Ioo des digesta collectés en $\mathrm{I}_{5}$ à $60 \mathrm{mn}$ ).

Compte tenu de tous ces aspects, un appareillage utilisable pour les contenus duodéno-jéjunaux du Porc a été réalisé.

\section{II. - Développement d'un système automatisé à l'usage des contenus digestifs du porc}

\section{I. - Recherche des principes de fonctionnement}

Le problème essentiel rencontré dans nos conditions avec un appareil identique à celui de Thivend et Poncet (I97I) réside, compte tenu des caractéristiques physiques des aliments et des contenus de duodénum du Porc, dans la décantation 


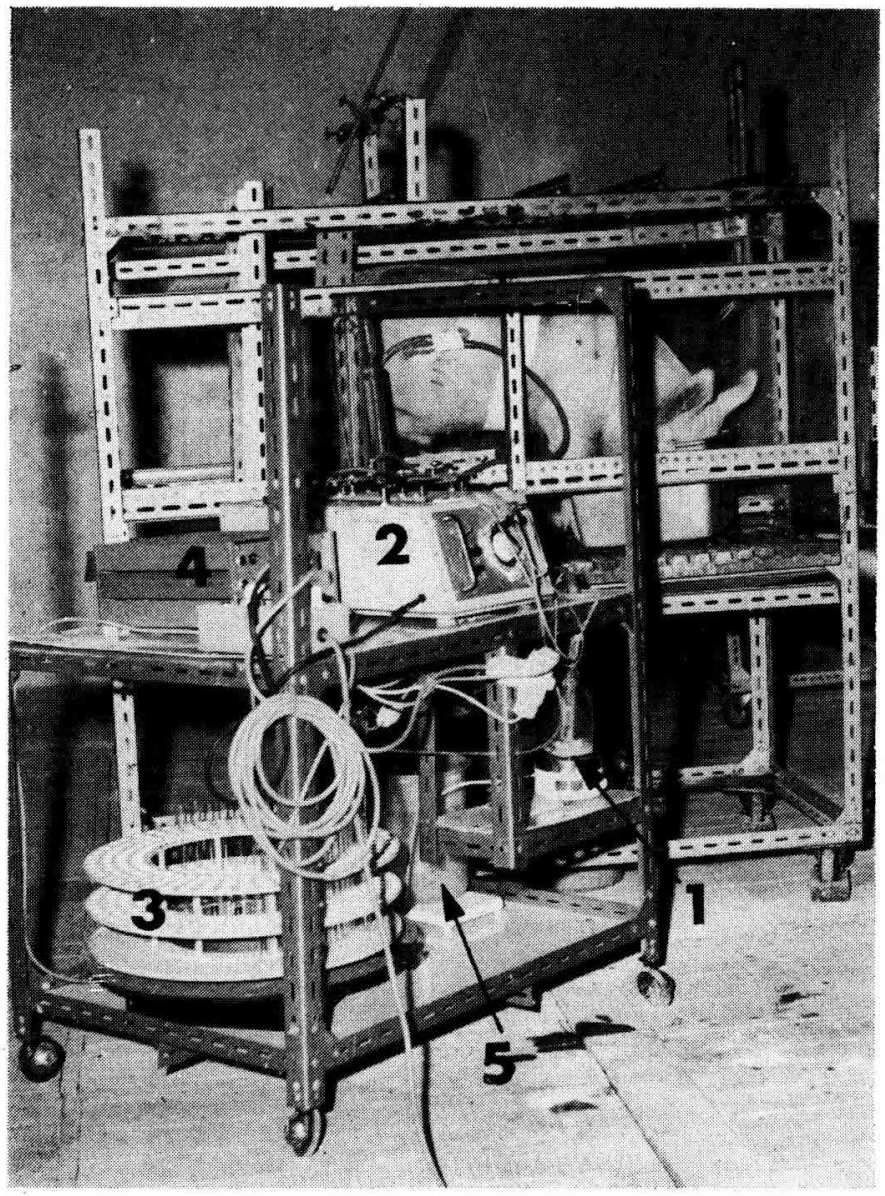

FIG. I. - Dispositif utilisé pour l'étude des principes de fonctionnement d'un système de collecte automatisé des contenus digestits de Porc.

I. Récipient d'homogénéisation des digesta émis, dans lequel s'effectuent les pompages.

2. Pompe proportionnante assurant le bullage à l'entrée des deux voies de pompage, le prélèvement d'une fraction aliquote $(5 \mathrm{p}$. Ioo) et la restitution des 95 p. I oo restants à l'animal.

3. Collecteur de fraction pour la collecte des fractions aliquotes.

4. Temporisation du collecteur.

5. Bain-marie alimentant le réchauffeur des digesta destinés à réintroduction.

Les éléments $n^{08} \mathrm{I}$ à 5 sont ceux que l'on retrouvera schématisés dans la figure 2 sous les sigles : $\mathrm{I}-\mathrm{AM} ; 2-\mathrm{PP} ; 3-\mathrm{CF} ; 4-\mathrm{PC} ; 5-\mathrm{BT}$.

$$
\begin{aligned}
& \text { Apparatus used to study the working principles } \\
& \text { of an automatic sampling system of digesta in the Pig. }
\end{aligned}
$$

I. Flask for homogenization of digesta outflow and pumping.

2. Proportioning pump for bubbling at the entry of the 2 pumping ways, sampling (5 per cent of total volume) and returning (95 per cent) to the animal.

3. Fraction collector to collect separately each aliquot (5 per cent) sample.

4. Timing of the fraction collector.

5. Heating bath to serve the heater of digesta flowing back to the pig.

Components $\mathrm{r}$ to 5 will be found in figure 2 as: $1-\mathrm{AM} ; 2-\mathrm{PP} ; 3-\mathrm{CF} ; 4-\mathrm{PC} ; 5-\mathrm{BT}$. 
qui survient dans les godets du tambour rotatif de collecte. Cette décantation est d'autant plus intense que l'évacuation des digesta est plus lente et par conséquent la rotation du tambour moins fréquente. Ces difficultés impliquent d'avoir recours à une agitation continue des digesta recueillis et à un pompage de la suspension ainsi entretenue, en accord avec AXFORD, Evans et OFFER (I97I). Leur technique prévoit cependant l'inversion périodique du sens de pompage pour lutter contre les blocages de la pompe. Face à ce problème persistant, nous avons choisi d'utiliser le principe d'un bullage régulier fractionnant la colonne fluide a fin de réduire le plus possible la séparation physique des particules et du liquide.

\section{TABLEAU I}

Echantillonnage par le système entièrement automatisé (pompe synchrone à triple fonction) : Comparaison de la composition d'échantillons obtenus automatiquement $(A)$ ou manuellement $(M) \dot{a}$ partir de digesta d'épreuve additionnés d'oxyde de chrome $\left(\mathrm{Cr}_{2} \mathrm{O}_{3}\right)$ adsorbé sur cellulose.

Sampling by the fully automatic system (synchronous three-function pump): Comparative composition of samples obtained either automatically $(A)$ or manually $(M)$ from test-digesta with an admixture of chromic oxyde $\left(\mathrm{Cr}_{2} \mathrm{O}_{3}\right)$ adsorbed on cellulose.

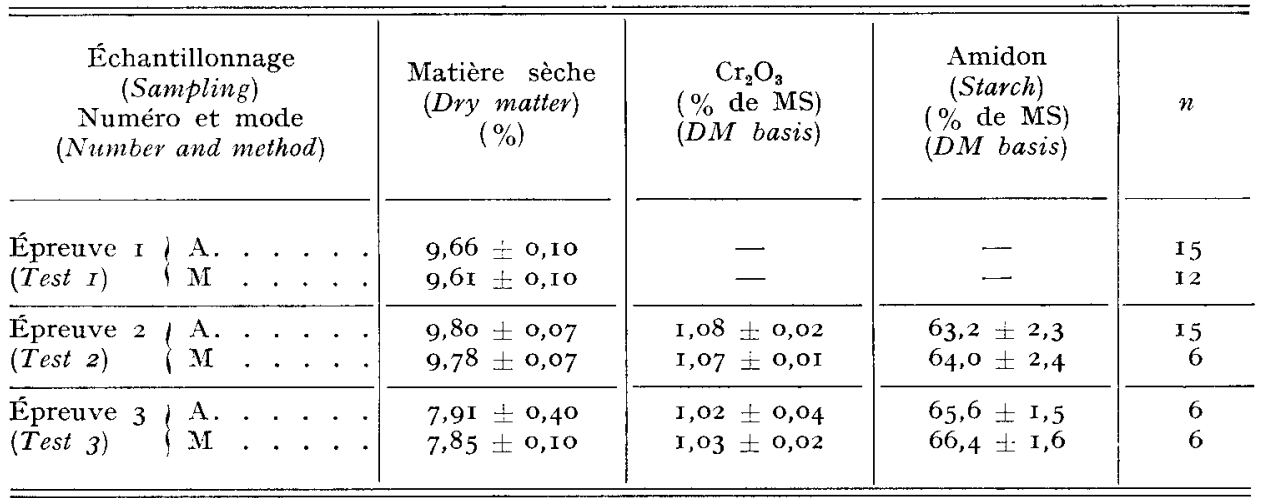

Sur ces bases (agitation continue, pompage avec bullage) un premier appareil a été élaboré (fig. I) qui comporte une pompe proportionnante assurant parallèlement, de façon synchrone, trois fonctions par des tubes de calibre différent : a) prélèvement de la fraction aliquote (5 p. IOO), b) restitution à l'animal (95 p. IOO) et c) bullage par 2 tubes distincts, proportionnellement aux débits à l'entrée des 2 autres voies. L'échantillon ainsi obtenu, et recueilli sur un collecteur de fractions en fonction du temps, présente une composition effectivement très proche de celle de la collecte totale dont il provient (tabl. I). Les digesta sont renvoyés à l'animal de façon continue, avec un faible délai (temps de franchissement des tubes de 30 à $50 \mathrm{sec}$.), au travers d'un manchon chauffant thermostaté $\left(39^{\circ} \mathrm{C}\right)$. La réintroduction dans l'intestin s'effectue par gravité à partir d'une ampoule ouverte à l'air libre dans laquelle se déversent les digesta pompés.

L'utilisation de cet appareillage pour la mesure du transit de repas d'épreuve nous a cependant montré la médiocrité des mesures de volume. Celles-ci reposent sur la seule lecture du volume de la fraction aliquote, rendue délicate et imprécise en raison de l'opacité du produit. 


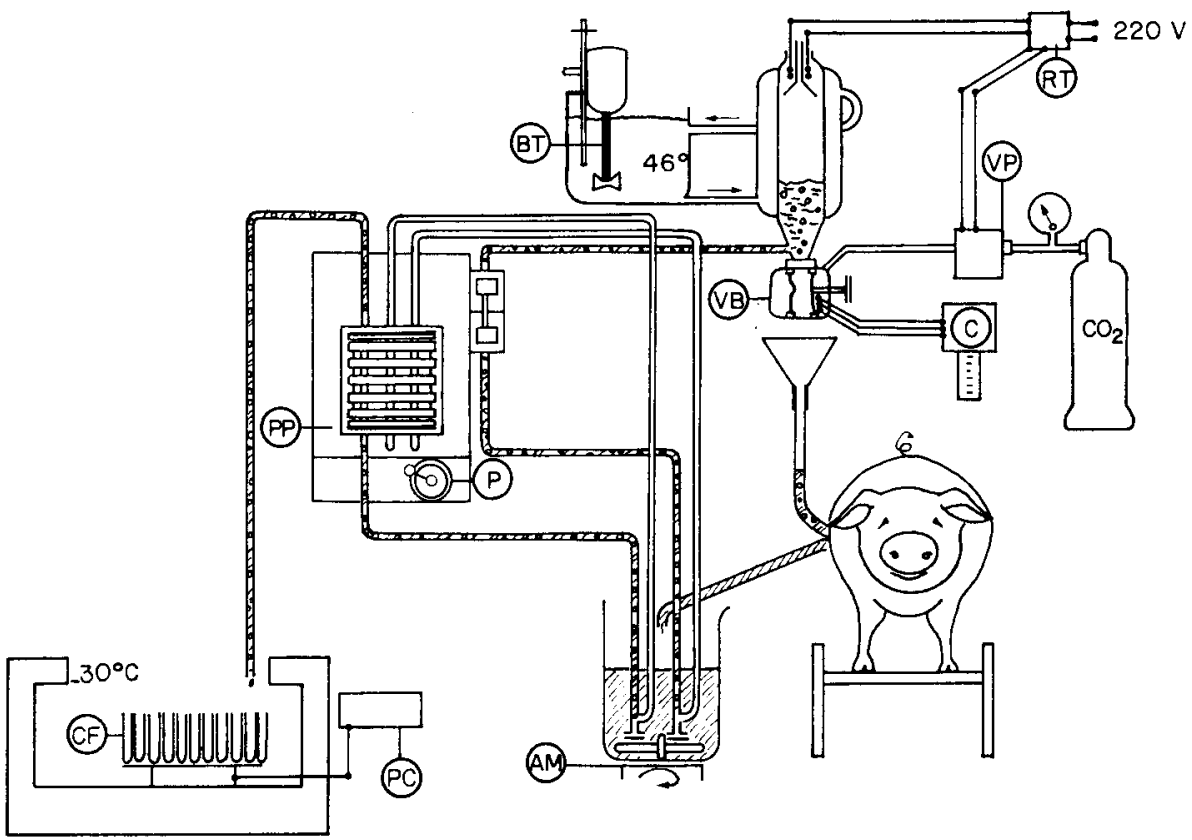

Frg, 2. - Schéma du dispositif utilisé pour l'automatisation complète de la collecte, de l'échantillonnage et de la véintroduction des contenus digestifs chez le Porc. Cet appareillage constitue dans la pratique un perfectionnement de celui illustré par la figure $I$, au niveau du contrôle de la réintroduction.

AM : Agitateur magnétique assurant l'homogénéisation des digesta émis.

PP : Pompe proportionnante ( 2 voies de bullage et 2 voies de pompage) à débit variable par l'intermédiaire d'un potentiomètre $(\mathrm{P})$.

$-30^{\circ} \mathrm{C}:$ Enceinte réfrigérée à $-3^{\circ} \mathrm{C}$ pour assurer le refroidissement très rapide des fractions aliquotes recueillies sur le collecteur de fractions (CF) dont la rotation est commandée à partir du boîtier pilote (PC).

BT : Bain thermostaté alimentant le réchauffeur des digesta restitués à l'animal.

VB : Vanne biologique à commande électropneumatique impliquant une vanne pilote

(VP) et un relais temporisateur (RT) commandant la fermeture de la vanne biologique au bout d'un temps réglable après l'ouverture déclenchée par les contacts plongeurs.

C : Compteur imprimant à horloge, enregistrant les ouvertures de la vanne et leur chronologie.

Scheme of the apparatus used for a fully automatic collecting, sampling and retuming of digesta in the Pig. This apparatus is analogous to that seen in figure $I$, after addition of the reintroduction control system.

AM : Magnetic stirrer to homogenize the digesta outflow.

PP : Proportioning pump ( 2 ways for bubbling and 2 ways for pumping) with adjustable flow by potentiometer (P).

$-30^{\circ} \mathrm{C}$ : Freezing unit to get a very quick fall of the temperature of the aliquot samples (CF) Fraction collector control unit (PC).

BT : Thermostatic heating bath to serve the heater of digesta flowing back to the Pig.

VB : Electromagnetic sluice-valve, with a pilot valve (VP) and a delaying-relay (RT) to control the duration of the opening of the sluice valve starting from the immersion of the electrodes.

C : Writing counter with a clock to record the opening of the sluice valve and its chronology. 


\section{2. - Automatisation complete}

L'application des principes retenus précédemment a guidé la réalisation de l'appareillage automatique schématisé dans la figure 2 . Le système utilisé comporte en particulier la mise en œuvre d'une mesure des volumes restitués à l'animal. Cette mesure est obtenue en plaçant à la base du réchauffeur une vanne à voie large (diam. int. $22 \mathrm{~mm}$ ) et passage direct, dont le fonctionnement est commandé pneumatiquement par une électrovanne pilote. L'ouverture est déclenchée par des contacts plongeurs lorsque le volume maximum admis (ajustable de 20 à $100 \mathrm{ml}$ ) est atteint. La fermeture est tributaire du réglage d'un relais temporisateur. Cet ensemble permet de libérer les digesta par fractions constantes ( $27 \mathrm{ml}$ en $0,5 \mathrm{sec}$.). La fréquence des ouvertures est enregistrée par période de Io mn sur une imprimante à horloge. Aucune décantation au-dessus de la vanne pneumatique n'a été observée grâce à l'agitation liée au bullage dans la tubulure de retour à l'animal. Enfin, à titre complémentaire, il faut mentionner l'usage d'une enceinte réfrigérée à $-30^{\circ} \mathrm{C}$ dans laquelle est placé le collecteur des fractions aliquotes a fin d'assurer un refroidissement très rapide des digesta destinés à analyse.

Ainsi complété, cet appareillage autorise deux contrôles volumétriques complémentaires : de la fraction aliquote d'une part (5 p. roo théoriques) et de la fraction restituée au porc d'autre part (95 p. Ioo théoriques). Mais l'absence de tout système mécanique alternatif ou intermittent, obtenue par la continuité des 3 fonctions de pompage, permet d'avoir une totale simultanéité de la collecte proprement dite, de l'échantillonnage, et de la réintroduction.

\section{3. - Précision et inconvénients de l'automatisme total}

La précision du système a été testée par pompage et échantillonnage " in vitro " d'un mélange alimentaire ou d'un contenu stomacal prélevé antérieurement, additionné d'oxyde de chrome $\left(\mathrm{Cr}_{2} \mathrm{O}_{3}\right)$ adsorbé sur poudre de cellulose (TISSERAND, Coleou et ZELTER, I962). Ces essais ont été réalisés pour diverses conditions de débit de pompe afin de vérifier la non séparation des constituants à étudier dans l'appareillage. Les échantillons aliquotes prélevés sur le collecteur de fractions ont fait individuellement l'objet d'une première estimation de la matière sèche par lyophilisation. Les échantillons regroupant, pour un intervalle de temps plus long, les prises aliquotes initiales, ont fait l'objet ensuite des dosages suivants : matière sèche $\left(24 \mathrm{~h}\right.$ à $\left.7{ }^{\circ}{ }^{\circ} \mathrm{C}\right)$, amidon selon EwERS (I972) et $\mathrm{Cr}_{2} \mathrm{O}_{3}$ selon STEveNSON et CLARE (I962). Les résultats des divers dosages effectués sur les fractions aliquotes sont étroitement corrélés avec ceux réalisés sur le mélange d'épreuve initial (tabl. I).

Cependant, cette bonne estimation de la teneur des contenus digestifs en leurs divers constituants, à partir des fractions aliquotes, se trouve entachée de deux défauts qui ont pu être constatés à l'occasion d'enregistrements effectués chez 5 porcs préparés selon LAPLACE et TOMASSONE (I970) : l'un concerne la rigueur de l'échantillonnage; l'autre tient à la précision des mesures de volume.

Dans l'appareillage décrit, la continuité du pompage permettrait, en théorie, d'assurer le fractionnement de chaque jet de contenu digestif sans délai et sans qu'intervienne un mélange avec l'écoulement immédiatement consécutif. Le pompage éventuel d'air entre deux évacuations de digesta ne pourrait provoquer aucune perturbation compte tenu de la conception du système. Mais il est exclu 
dans la pratique d'atteindre ce système "idéal " en raison de la nécessité de l'agitation continue évitant toute décantation nuisible tant mécaniquement que pour la qualité d'échantillonnage. De ce fait le récipient de collecte, dans lequel s'effectuent agitation et aspiration par les tubes de pompe, comporte nécessairement un volume mort minimum nuisant à la rigueur de l'échantillonnage.

La précision relative des mesures de volume obtenues dans le cadre d'un tel enregistrement automatique peut être estimée par le coefficient de variation de la pente de la droite de régression entre les volumes libérés par la vanne électropneumatique à la réintroduction et les volumes fournis par un contrôle manuel intermédiaire à la sortie de la canule. Cette précision reste modérée puisque supérieure à 5 p. IOO (tabl. 2).

\section{TABLEAU 2}

Covrélation entre les mesures manuelle et automatique

du volume des effuents gastro-duodénaux au niveau d'une canule duodénale proximale $x=$ nombre de volumes de digesta libérés par l'électrovanne à la réintroduction.

$y=$ volume de digesta émis au niveau de la canule, mesuré à l'éprouvette.

\section{Correlation between manual and automatic measurements}

of the volume of digesta flowing through a duodenal fistula

$x=$ number of volume-units of digesta released by the electropneumatic sluice-valve for reintroduction.

$y=$ volume of digesta collected at the proximal cannula.

\begin{tabular}{|c|c|c|c|c|c|}
\hline $\begin{array}{l}\text { Essai } \\
(T e s t)\end{array}$ & $\begin{array}{c}\text { Nombre de } \\
\text { mesures } \\
\text { (Number of } \\
\text { measure- } \\
\text { ments) }\end{array}$ & $\begin{array}{l}\text { Coefficient } \mathrm{de} \\
\text { corrélation. } \\
\text { (Correlation } \\
\text { coefficient) }\end{array}$ & $\begin{array}{l}\text { Eqquation de régression } \\
\qquad y=b x+a\end{array}$ & $s_{b}$ & $\mathbf{P}<$ \\
\hline I & 42 & 0,99 & $y=27,62 x+1680,3$ & 0,8 & $0, \mathrm{or}$ \\
\hline 2 & 20 & 0,99 & $y=16,26 x+378,8$ & 0,3 & 0,01 \\
\hline 3 & 22 & 0,99 & $y=22,24 x+1076,4$ & 0,5 & $0,0 \mathrm{I}$ \\
\hline
\end{tabular}

$s_{b}=$ écart-type de la population des coefficients $b$ pour chaque couple de données. standard deviation of $b$ coefficients for each pair of data.

Enfin, les principales sources d'échec ou d'incidents ont toujours été le fait des porcs dont le caractère très turbulent dans une situation expérimentale exige une surveillance permancnte. Il est en particulier nécessaire de contrôler la position de l'animal : le décubitus latéral complet sur le flanc fistulé conduit à l'arrachement ou à l'écrasement des tubes. Les divers systèmes de suspension ou balanciers ne peuvent limiter correctement les mouvements provoqués des tubulures. Pour cette raison, l'appareillage a été remanié dans le sens d'une semi-automatisation.

\section{4. - Semi-automatisation}

Les modifications apportées dans le cadre d'un fonctionnement semi-automatique concernent d'une part la collecte proprement dite, d'autre part le pompage. comme le schématise la figure 3 . 
Les effluents gastro-duodénaux sont collectés pour des intervalles de $5 \mathrm{mn}$ au cours de la première heure post-prandiale, ou de Io mn ultérieurement, dans une éprouvette graduée ce qui fournit une mesure du volume émis à la canule proximale avec une précision de l'ordre de $2 \mathrm{p}$. Ioo seulement. Le contenu de l'éprouvette est transféré manuellement dans le récipient d'homogénéisation (agitateur magnétique) où s'effectue une reprise totale par les tubes de pompe avec bullage selon le principe précédemment décrit. Ce processus permet, à la différence du système automatique, d'assurer que les digesta soumis au fractionnement (5-95 p. Ioo) sont exclusivement ceux émis au cours de l'unité de temps considérée sans possibilité de mélange. On peut donc dans ce cas être certain d'avoir un échantillon représentatif du contenu recueilli pendant la période correspondant au volume prélevé.

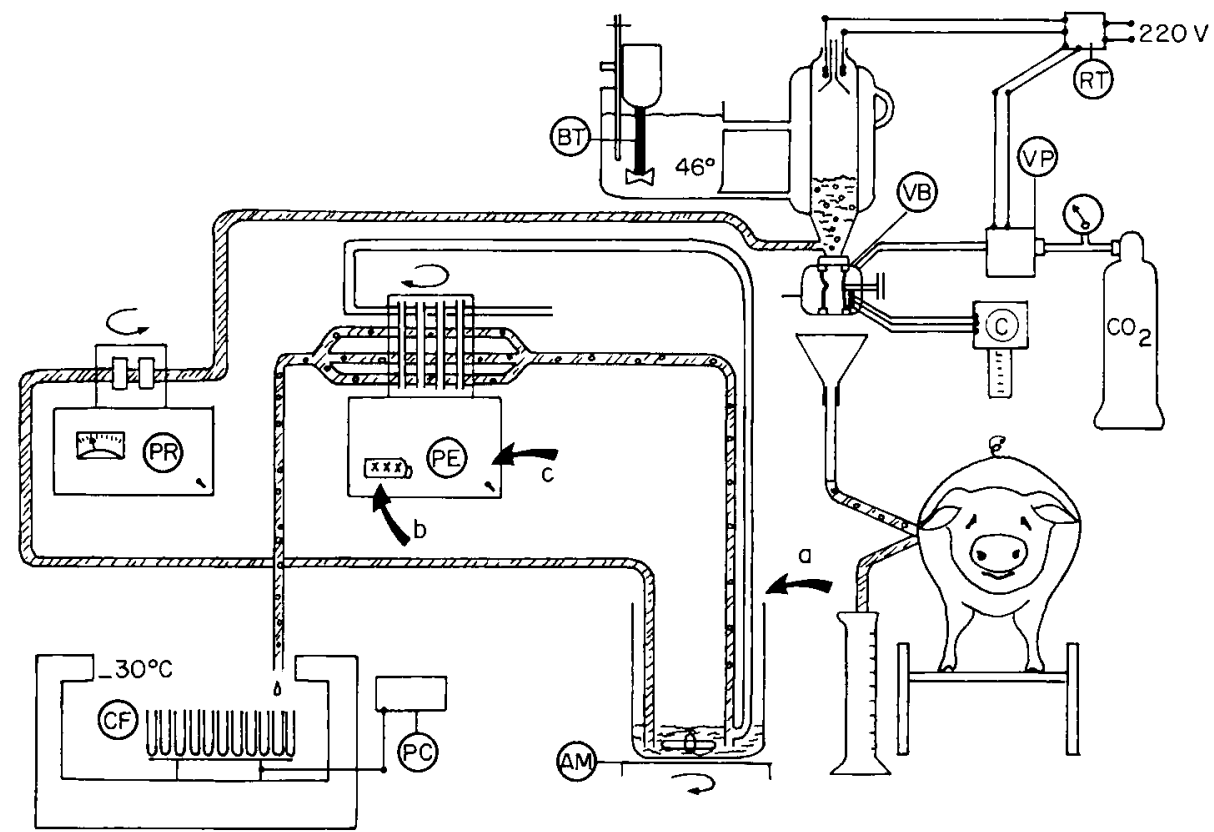

FIG. 3. - Schéma du dispositif semi-automatique de fractionnement des contenus digistits de Porc. Les flèches $a, b, c$ illustrent les actions manuelles maintenues :

a. : Transvasement du volume de digesta collecté dans l'éprouvette par unité de temps.

b : Affichage du débit de la pompe échantillonneuse, choisi d'après une abaque en fonction du volume recueilli en a.

c : Mise en fonction de la pompe échantillonncuse pendant un temps constant.

Toutes les abréviations sont identiques à celles utilisées dans lá figure 2 à l'exception de:

PR : Pompe assurant la restitution à l'animal de 95 p. 100 des digesta.

$\mathrm{PE}$ : Pompe échantillonneuse assurant le prélèvement d'une fraction aliquote de $5 \mathrm{p}$. 1 oo et le bullage à l'entréc de la tubulure de prélèvement.

Scheme of the semi automated apparatus used to partition digesta of the Pig. The arrows $a, b, c$ show the manual actions:

a : Transfer of the volume of digesta collected within a testglass during a time unit.

b : Determination of the flow rate of the sampling pump as a function of the collected volume.

c : Turning on the sampling pump during a constant time period.

All abbreviations are identical to that used in the figure 2 excepted:

PR : Pump for returning 95 per cent of digesta to the pig.

PE : Pump for sampling an aliquot (5 per cent) and bubbling at the opening of the sampling tube. 
Mais cet avantage est obtenu en renonçant à la continuité et à la simultanéité des opérations au profit de la temporisation imposée par la collecte (fractions de 5 à Io $\mathrm{mn}$ ).

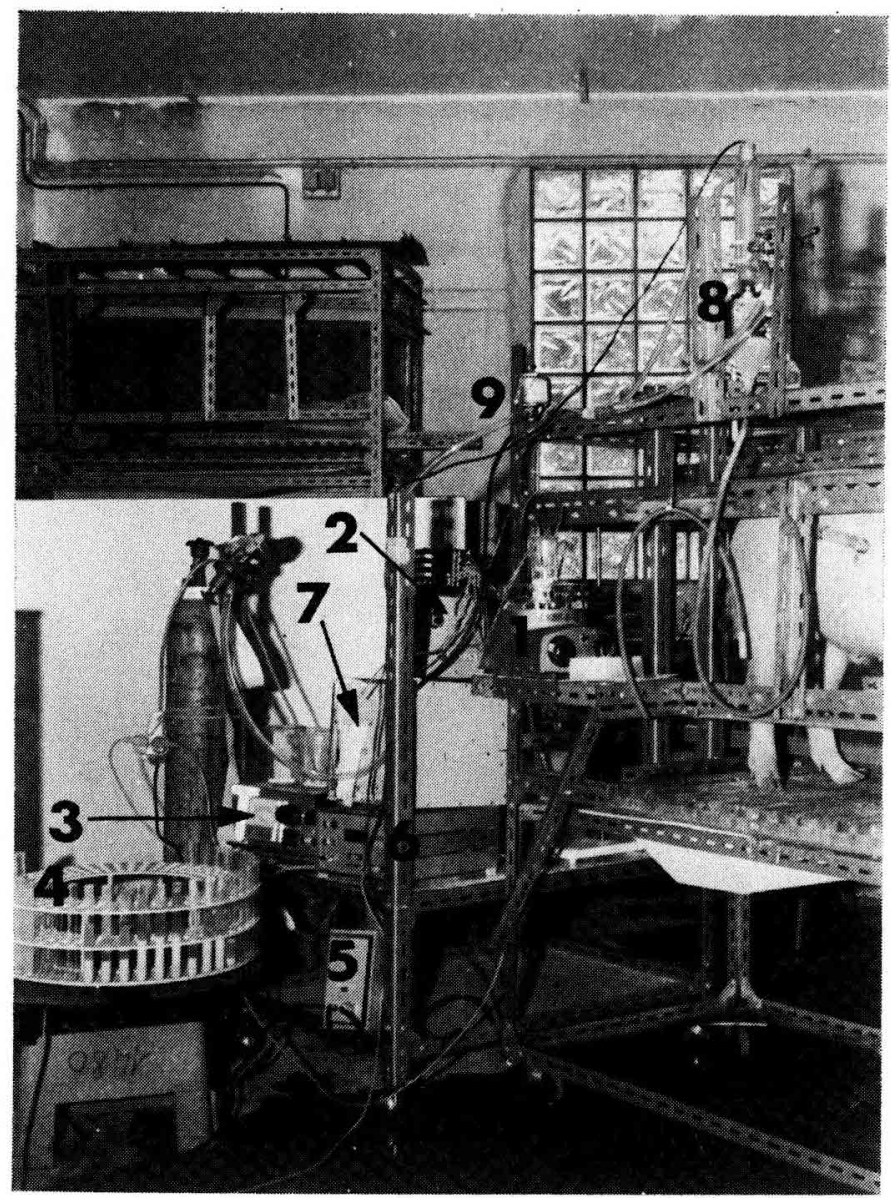

FIG. 4. - Dispositif semi-automatique de fractionnement des contenus digestifs du Porc, tel qu'il a été mis en cuvre au Laboratoive, à l'exception de l'enceinte véfvigévée exclue pour la photographie en raison de son encombroment. Le principe en est décrit par la figure $3: I=A M$, $2=P E, 3=P R, 4=C F, 5=R T, 6=P C, 7=C, 8=V B, 9=V P$.

Semi automatic device to partition digesta of the Pig, as used at the laboratory, freesing unit excepted not used for pholography because it was too large. The components are schematized in figuve 3 : $I=A M, 2=P E, 3=P R, 4=C F, 5=R T, 6=P C, 7=C, 8=V B, 9=V P$.

La modification des opérations de pompage consiste à la fois en l'adoption d'un pompage séparé des 2 fractions ( 5 et 95 p. Ioo) et en la commande manuelle de l'un des inducteurs de débit. Le prélèvement de l'échantillon est réalisé par une pompe Minipuls II Gilson HP4, équipée de 4 tubes de même calibre (2,79 mm int.). L'un assure l'injection d'air par un dispositif en forme de " $\mathrm{h}$ " (Technicon $\mathrm{R}$ ) à l'entrée de l'aspiration des jus intestinaux. Les trois autres sont destinés au prélèvement de l'échantillon. L'opérateur commande le fonctionnement de cette pompe 


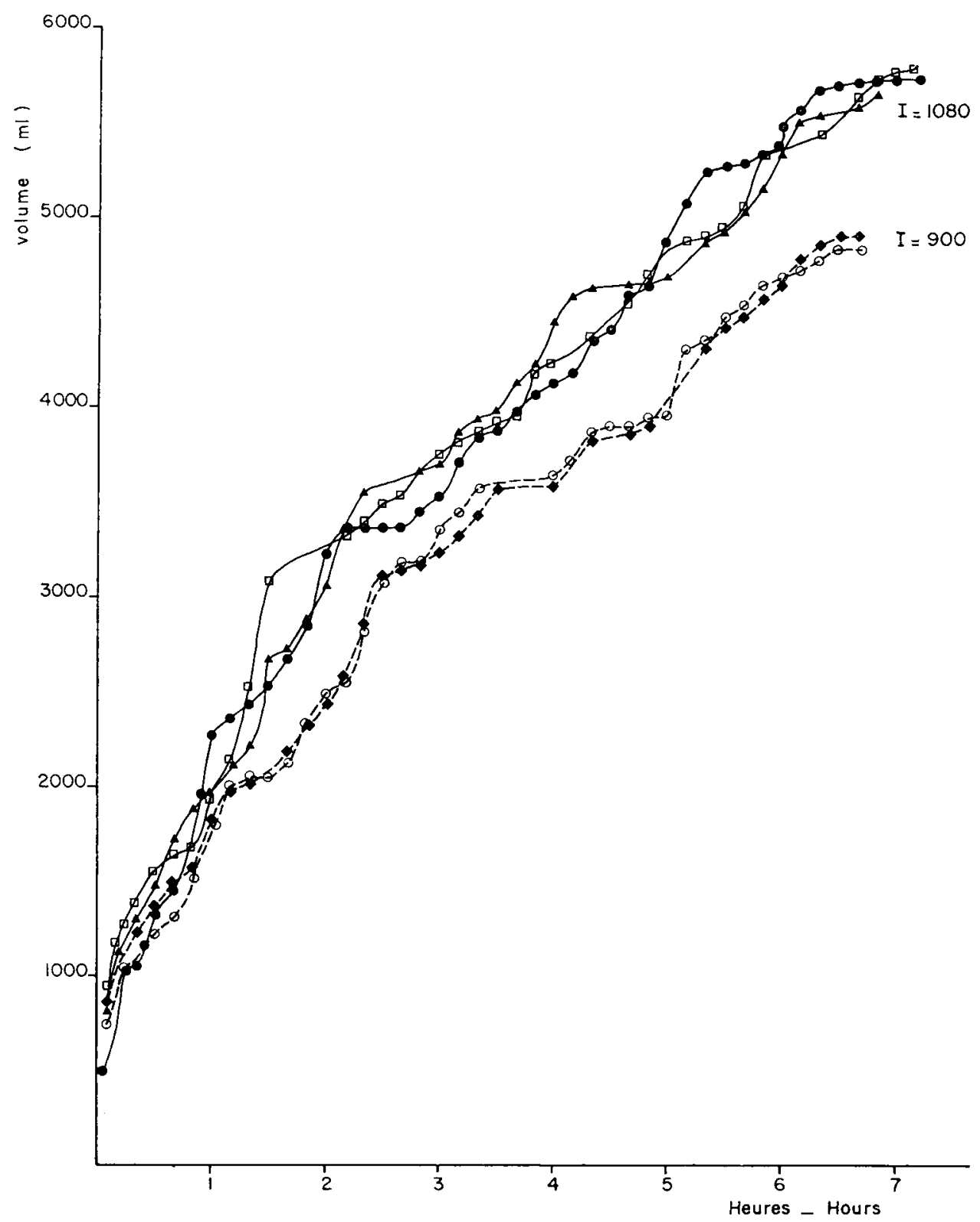

FIG. 5. - Volume cumulé des digesta émis au niveau d'une canule duodénale proximale chez un même porc $(55 \mathrm{~kg})$ après ingestion, le matin à $9 \mathrm{~h}$, d'un aliment purifiè à base d'amidon de maïs.

- Tracés continus : repas d'épreuve de $\mathrm{I}$ o8o $\mathrm{g}$ de matière sèche (3 essais).

- Tracés pointillés : repas d'épreuve de $900 \mathrm{~g}$ de matière sèche (2 essais).

Lc poids total de matière fraîche ingérée est, compte tenu de la dilution, respectivement de $4200 \mathrm{~g}$ et $3500 \mathrm{~g}$.

Cumulated volume of digesta flowing from a proximal duodenal cannula. in the same pig $(55 \mathrm{~kg})$ after the morning meal $(9 \mathrm{a} . \mathrm{m})$ : purified diet with corn starch.

- Full lines: 3 test meals of $\mathrm{I}$ o80 $\mathrm{g}$ of dry matter.

- Dotted lines: 2 test meals of $900 \mathrm{~g}$ of dry matter.

The total wet weight of food ingested is $4,200 \mathrm{~g}$ and $3,500 \mathrm{~g}$ respectively (dilution included). 
pendant un temps constant de I mn à un débit affiché, déterminé par une abaque en fonction du volume mesuré. Ce contrôle permanent autorise une grande précision de l'échantillonnage (fraction aliquote de 5,0 p. IO0). Le pompage des 95 p. Ioo restants est assuré séparément par une pompe Transoméga IIA qui alimente le réservoir du réchauffeur au niveau duquel intervient automatiquement la temporisation et l'enregistrement des opérations de restitution à l'animal. La photogra-

\section{TABLEAU 3}

Échantillonnage par le système semi-automatique (pompes séparées pour échantillonnage et réintroduction) : Comparaison de la composition d'échantillons obtenus automatiquement $(A)$ ou manuellement $(M)$ à partir de digesta d'épreuve additionnés de polyéthylène glycol (PEG) et d'oxyde. de chrome $\left(\mathrm{Cr}_{2} \mathrm{O}_{3}\right)$ adsorbé sur cellulose.

Sampling by the semi-automatic system (separate pumps for sampling and reintroduction) : Comparative composition of samples obtained either automatically $(A)$ or manually $(M)$ from testdigesta with an admixture of polyethylene glycol $(\mathrm{PEG})$ and chromic oxyde $\left(\mathrm{C}_{2} \mathrm{O}_{3}\right)$ adsorbed on cellulose.

\begin{tabular}{|c|c|c|c|c|c|}
\hline $\begin{array}{c}\text { Échantillonnage } \\
\text { (Sampling) } \\
\text { Numéro et mode } \\
\text { (Number and } \\
\text { method) }\end{array}$ & $\begin{array}{c}\text { Matière sèche } \\
(\text { Dry matter }) \\
(\%)\end{array}$ & $\begin{array}{c}\mathrm{Cr}_{2} \mathrm{O}_{3} \\
(\% \text { de MS }) \\
(D M \text { basis })\end{array}$ & $\begin{array}{c}\text { PEG } \\
(\% \text { de MS }) \\
(D M \text { basis })\end{array}$ & $\begin{array}{c}\text { Amidon } \\
\text { (Starch) } \\
(\% \text { de MS }) \\
(D M \text { basis })\end{array}$ & $n$ \\
\hline \begin{tabular}{l|l} 
Eppreuve I & $\mathrm{A}$ \\
$($ Test I & M
\end{tabular} & $\begin{array}{l}8,89 \pm 0,10 \\
8,88 \pm 0,09\end{array}$ & $\begin{array}{l}0,72 \pm 0,05 \\
0,75 \pm 0,06\end{array}$ & $\begin{array}{l}\mathrm{I}, 72 \pm 0,32 \\
\mathrm{I}, 74 \pm 0,25\end{array}$ & $\begin{array}{l}66,5 \pm 0,9 \\
66,3 \pm 0,8\end{array}$ & $\begin{array}{l}4 \\
6\end{array}$ \\
\hline \begin{tabular}{l|l} 
Epreuve 2 & $\mathrm{~A}$ \\
$($ Test 2) & $\mathrm{M}$
\end{tabular} & $\begin{array}{l}7,95 \pm 0,34 \\
8,05 \pm 0,08\end{array}$ & $\begin{array}{l}0,70 \pm 0,12 \\
0,71 \pm 0,07\end{array}$ & $\begin{array}{l}\mathbf{1}, 77 \pm 0,40 \\
\mathbf{I}, 81 \pm 0,15\end{array}$ & $\begin{array}{l}64,8 \pm \mathrm{I}, \mathrm{I} \\
65,3 \pm 0,8\end{array}$ & $\begin{array}{r}10 \\
9\end{array}$ \\
\hline $\begin{array}{l}\text { Epreuve } 3 \\
\text { (Test 3) }\end{array}$ & $\begin{array}{l}7,42 \pm 0,10 \\
7,4^{\circ} \pm 0,16\end{array}$ & $\begin{array}{l}0,80 \pm 0,03 \\
0,74 \pm 0,09\end{array}$ & $\begin{array}{l}1,81 \pm 0,09 \\
1,70 \pm 0,28\end{array}$ & $\begin{array}{l}66,6 \pm 0,6 \\
65,6 \pm 1,0\end{array}$ & $\begin{array}{l}9 \\
9\end{array}$ \\
\hline
\end{tabular}

phie de l'appareillage (fig. 4) illustre la complexité et l'encombrement actuels du système.

Dans ces conditions, les trois mesures de volume, réalisées à la collecte, à l'échantillonnage et à la réintroduction, fournissent des résultats convergents d'une précision meilleure ( 2 à $3 \mathrm{p}$. IOo) que celle fournie par le système entièrement automatique. Les données ainsi obtenues en $8 \mathrm{~h}$ à la suite de l'ingestion d'un aliment purifié, comportant notamment Io,4 p. Ioo de caséine chlorhydrique et 67,5 p. Ioo d'amidon de maïs, dilué par 2,5 fois son poids d'eau, sont présentées dans la figure 5. La qualité de l'échantillonnage assuré par ce système semi-automatique a été évaluée (tabl. 3) de la même façon que précédemment pour l'appareillage automatique, par dosage de la matière sèche, de l'amidon, du $\mathrm{Cr}_{2} \mathrm{O}_{3}$, ainsi que du polyéthylène glycol (PEG 400o) selon Sмiтн (I959). En définitive, la précision d'estimation de la teneur en amidon atteint I,5 p. Ioo (coefficient de variation sur Io répétitions de trois échantillonnages du mélange d'épreuve et du prélèvement aliquote). Elle est de $\mathrm{I}, 7$ p. Ioo pour $\mathrm{Cr}_{2} \mathrm{O}_{3}$, de 7,7 p. Ioo pour le PEG et de 6,o p. roo pour la matière sèche. 


\section{III. - Discussion et conclusion}

A la lumière des essais et expérimentations réalisés chez le Porc, avec l'un ou l'autre des systèmes décrits, on peut résumer leurs avantages et inconvénients spécifiques comme suit :

- l'automatisation totale garantit une simultanéité de la collecte, de l'échantillonnage, et de la réintroduction, sans délai autre que le temps, très faible, de franchissement des tubulures. Mais, indépendamment du fait qu'une surveillance étroite reste nécessaire en raison du comportement des animaux, la rigueur de l'échantillonnage n'est pas absolue et la précision des mesures de volume reste modérée.

- la semi-automatisation assure une parfaite représentativité de l'échantillonnage et une précision rigoureuse du volume de cette fraction aliquote, qualités particulièrement appréciables pour une étude cinétique de la digestion. Le contrôle humain, de toute façon nécessaire, fournit aussi une utile lecture de volume préalable à toute séparation. Le procédé introduit une temporisation (5 à Io mn) qui permet en définitive d'obtenir les qualités précitées de l'échantillon.

Si l'automatisme complet semble mieux respecter la fonction digestive en l'absence de toute temporisation, il faut reconnaître que, en dépit du décalage constant, le rythme de restitution réalisé dans le système semi-automatique reste aussi proche que possible du rythme d'émission à la canule. Aussi en dépit de cet inconvénient, mineur en raison de la régularité des opérations, le système semiautomatique paraît le mieux adapté à toute étude de digestion chez le Porc en raison de la meilleure précision (de l'ordre de 2 à 3 p. Ioo) des mesures de volume et de la représentativité de l'échantillonnage, ce qui éviterait un recours systématique à une correction par des marqueurs.

Néanmoins, il serait prématuré au terme de ce travail, de porter un jugement définitif affirmant la supériorité du système semi-automatique décrit. Sur un plan général, il faut souligner que le système proposé n'est pas actuellement applicable aux contenus de l'iléon du Porc, compte tenu des problèmes rencontrés à ce niveau (LAPLACE et Borgida, I976). Ce cas particulier devra être réexaminé en relation avec l'application de la technique de fistulation proposée par Ivan (I974). A noter que l'utilisation faite par ce dernier (Ivan et BOwLAND, I976) de cette fistulation iléale consiste en une collecte par périodes de 60 à i $20 \mathrm{mn}$ ce qui suppose donc une restitution différée d'autant. Il est vrai que l'importance des conditions de rythme et de volume à la réintroduction est très différente pour l'intestin grêle terminal de ce qu'elle est au niveau du duodénum proximal, point de départ d'une intéroception particulièrement riche.

D'un point de vue plus technique, on peut s'interroger sur l'éventualité d'une modification du transit digestif lors de mise en ouvre de ce système : cette modification est-elle précoce mais transitoire, ou à l'inverse différée et liée à un usage prolongé? La question est d'importance chez le Porc à l'égard des temps d'évacuation gastro-duodénale d'un repas d'épreuve. Il sera également intéressant de tester le taux de récupération d'un marqueur ingéré dans l'aliment. On pourra aussi examiner des aspects de moindre importance tels que effet de la réintroduction par simple gravité, ou incidence du bullage (serait-il à l'azote).

Plutôt que de figer l'appareillage décrit en multipliant les études méthodologiques à son sujet, il paraît opportun d'en envisager dès à présent l'amélioration. La récente mise au point d'un débitmètre électromagnétique spécialement adapté aux débits de contenus digestifs (PoNCET et al., 1977) pourrait en être l'instrument. 
Cette technique de débitmétrie est particulièrement adaptée à des études de physiologie du débit de digesta en relation par exemple avec des phénomènes moteurs et à l'exclusion de toute biochimie des contenus. Elle peut éventuellement chez les Ruminants autoriser une étude de bilan digestif établi sur plusieurs jours avec des prélèvements discontinus. Ce dernier procédé étant inadéquat chez le Porc, on ne peut décrire par ce moyen la cinétique de transformation des matériaux alimentaires et de disparition des produits de la digestion, corollaire de la cinétique d'absorption des substances apparaissant dans le sang portal (RERAT, I975). Par contre, on peut tenter de réunir les avantages de la collecte totale selon un système plus ou moins automatisé et ceux de la débitmétrie électromagnétique.

Dans ce sens a été envisagée (Thivend, communication personnelle I977) l'association chez le Mouton de l'appareillage portable de TAYlor, Weller et REID (I97I) et du débitmètre électromagnétique (PonCET et al., I977) pour parvenir à un échantillonnage plus représentatif. Dans le cas de l'appareillage semiautomatique décrit plus haut à l'usage du Porc, la lecture effectuée au niveau du récipient de collecte manuelle pourrait être remplacée par l'enregistrement du débit immédiatement en amont de la canule ou sur la canule proximale elle-même. Cet enregistrement quantité/temps/débit pourrait aisément déclencher le fonctionnement de la pompe d'échantillonnage en déterminant aussi son débit pour un calibre standard de tube. Enfin ces mêmes informations mémorisées pourraient servir à réguler la réintroduction selon un modèle fidèle à l'émission des contenus.

$E n$ conclusion, la collecte totale des digesta chez l'animal équipé d'une fistule réentrante s'avère préférable à toute autre technique chez le Porc pour une étude cinétique de la digestion. En l'état actuel des travaux, c'est par une automatisation partielle des manœuvres qu'est obtenu le meilleur échantillonnage des digesta et non dans le cadre d'un système entièrement automatique. En lui-même perfectible et devant faire l'objet de diverses vérifications méthodologiques, le système proposé devrait cependant évoluer vers un automatisme plus complet par asservissement de diverses manœuvres à une mesure électromagnétique du débit des digesta.

Accepté pour publication en mai 1977.

\section{Summary}

\section{Total collection of intestinal contents in the Pig. Automatic sampling and restitution of digesta to the animal for kinetic studies of digestion}

An analysis is made of the potential interest of developing an automatic device for total collection, sampling and restitution of digesta in monogastric animals and more especially in pigs. A bibliographical study of methodological works made in ruminants was carried out in order to estimate the most fitted technique for pigs (total collection or use of markers) on account of the physiological and behavioural particuliarities of these animals.

The working principles of an automatic sampling system of digesta were determined and a full-automatic apparatus was developed. After having analysed experimentally the specific advantages and disadvantages of this apparatus, a semi-automatic system, better adapted to recordings in pigs, was elaborated. Contrarily to the full automatization, this semi-automatic device did not lead to a perfect simultaneousness in the collection, sampling and reintroduction of digesta. However, the samples obtained were perfectly representative, and the measurements of volumes more accurate (2-3 per cent more) leading to a better estimation of the digestion of such and such dietary constituent.

The systems described can obviously be further improved. The possibility of using an electromagnetic device (digesta flow measurement) for controlling an automatic apparatus is considered. 


\section{Références bibliographiques}

Auffray P., Martinet J., Rerat A., 1967. Quelques aspects du transit gastro-intestinal chez le Porc. Ann. Biol. anim. Bioch. Biophys., 7, 261-279.

AXFord R. F. E., Evans R. A., OFFer N. W., I97I. An automatic device for sampling digesta from the duodenum of the sheep. Res. Vet. Sci., 12, I28-131.

Corse D. A., 1974. Automatic sampling of digesta. Proc. Nutr. Soc., 33, I 4 I-I 46.

CoRse D. A., Sutton J. D., I97I. Methods of measuring flow of duodenal digesta in sheep. Proc. Nutr. Soc., 30, I8A-19A.

Ehouinsou M. A., I976. Étude de la digestion du lactose chez le Mouton. Thèse Doct. Ing., Clermont-Ferrand, $90 \mathrm{p}$.

EvANs R. A., AXFord R. F. E., OFFER N. W., I97I. An automatic method for sampling digesta in vivo. Proc. Nutr. Soc., 30, 40A-4IA.

Ewers E., 1972. Dosage de l'amidon par la méthode polarimétrique. J. O. C.E.E., 15, L I23, 7-9.

FaIChNey G. J., I975. The use of markers to partition digestion within the gastro intestinal tract of ruminants. Pp 277 à 29r, in: M.c Donald I. W., Warner A. C. I., Digestion and Metabolism in the Ruminant, Proc. IV Int. Symp. Ruminant Physiol., Sydney.

Holmes J. H. G., Bayley H. S., Horney F. D., 1974. Digestion of dry and high moisture maize diets in the stomach of the pig. $B r . J$. Nutr., 32, 639-646.

Ivan M., I974. A new type of re-entrant cannula designed for use in the small intestine of the pig. Austral. Vet. $J, 50,547-552$.

Ivan M., Bowland J. P., 1976. Digestion of nutrients in the small intestine of pigs fed diets containing raw and autoclaved faba beans. Can. J. Anim. Sci., 56, 45I-456.

Котв A. R., Luckey T. D., I972. Markers in nutrition. Nutr. Abstr. Rev., 42, 81 3-845.

LAPLACE J. P., 1972. Le transit digestif chez les monogastriques. I) Les techniques d'étude. Ann. Zootech., 21, 83-105.

LAPLACE J. P., 1975. Le transit digestif chez les monogastriques. 2) Phénomènes moteurs et mouvements des digesta. Ann. Zootech., 24, 489-552.

Laplace J. P., Borgida L. P., 1976. Problèmes physiologiques posés par la fistulation réentrante chronique de l'iléon chez le Porc : Étude bibliographique et expérimentale. Ann. Zootech., 25, 36I-37I.

Laplace J. P., Lebas F., 1975. Le transit digestif chez le Lapin. 3) Influence de l'heure et du mode d'administration sur l'excrétion fécale du cérium-I $4^{I}$ chez le lapin alimenté ad libitum. Ann. Zootech., 24, 255-265.

Laplace J. P., Tomassone R., 1970. Evvacuation gastro-duodénale chez le Porc. Fistulation chronique par voie thoracique extra-pleurale; recherche d'une technique d'analyse mathématique de l'évacuation. Ann. Zootech., 19, 303-332.

MACRAE J. C., I975. The use of re-entrant cannulae to partition digestive function within the gastro intestinal tract of ruminants. p. 26r à 276, in: Mc Donald I. W., Warner A. C. I., Digestion and Metabolism in the Ruminant, Proc. IV Int. Symp. Ruminant Physiol., Sydney.

Poncet C., Dimova E., Leveille M., Dardillat C., I977. Enregistrement simultané chronique du comportement alimentaire, du débit duodénal et de l'électromyogramme gastro-intestinal. Ann. Biol. anim. Bioch. Biophys., 17, sous presse.

Rerat A., I975. Mesure quantitative in vivo de l'absorption chez le Porc : Application aux sucres et aux acides aminés. In: réanimation entérale à faible débit continu, I.N.S.E.R.M. ed., Colloque Paris 20 nov., 53, 47-62.

Rerat A., Corring T., Laplace J. P., 1976. Protein digestion and absorption, pp. 97-138, in Cole D. J. A., Boorman K. N., Butrery P. J., Lewis D., Neale R. J., Swan H., Protein Metabolism and Nutrition, chap. II, Digestion and availability, I vol., 5 I5 p., Butterworth. Ed., Londres.

Smity R. H., 1959. The development and function of rumen in milk fed calves. J. agric. Sci., 52, $72-78$.

Stevenson A. E., Clare N. T., 1962. Measurement of feed intake by grazing cattle and sheep. IX Determination of chromic oxide in faeces using an autoanalyser. N.Z. J. Agric. Res., 6, I2I-I 26.

Sutton J. D., Youssef F. G., Oldham J. D., 1976. Measurements over 5d of the flow of dry matter and of chromic oxide at the duodenum of cattle. Proc. Nutr. Soc., 35, IooA-rorA.

TAYlor M. N., Weller R. A., Reid C. S. W., I97I. A method for automatic sampling of digesta from the duodenum, in: Weller R. A., Prlgrim A. F., Gray F. V., Level of food intake and the passage of markers and nitrogen along the alimentary tract of sheep. $B r . J . N u t r ., 26$, $487-497$. 
Thivend P., Poncet C., I97 I. Prélèvement automatique de contenus intestinaux chez le Veau et le Mouton. Ann. Biol. anim. Bioch. Biophys., 11, 274-275.

Tisserand J. L., Coleou J., Zelrer S. Z., I962. Emploi du sesquioxyde de chrome comme indicateur dans les études de bilan digestif chez le Ruminant. C. R. Acad. Sci., Paris, 254, 2233-2235.

Zebrowska T., Buraczewska L., Buraczewski S., Horszczaruk F., 1975. Digestion and absorption in the small intestine of pigs. Part. I. Digestion and absorption of dry matter and nitrogen. Rocs. Nauk Roln., 96B, 79-90. 